\title{
Some extragradient methods for common solutions of generalized equilibrium problems and fixed points of nonexpansive mappings
}

Jian-Wen Peng

\author{
Correspondence: jwpeng6@yahoo. \\ com.cn \\ School of Mathematics, Chongqing \\ Normal University, Chongqing \\ 400047, PR China
}

\begin{abstract}
In this article, we introduce some new iterative schemes based on the extragradient method (and the hybrid method) for finding a common element of the set of solutions of a generalized equilibrium problem, and the set of fixed points of a family of infinitely nonexpansive mappings and the set of solutions of the variational inequality for a monotone, Lipschitz-continuous mapping in Hilbert spaces. We obtain some strong convergence theorems and weak convergence theorems. The results in this article generalize, improve, and unify some well-known convergence theorems in the literature.
\end{abstract}

Keywords: Generalized equilibrium problem, Extragradient method, Hybrid method, Nonex-pansive mapping, Strong convergence, Weak convergence

\section{Introduction}

Let $H$ be a real Hilbert space with inner product $\langle.,$.$\rangle and induced norm \|\cdot\|$. Let $C$ be a nonempty closed convex subset of $H$. Let $F$ be a bifunction from $C \times C$ to $R$ and let $B: C \rightarrow H$ be a nonlinear mapping, where $R$ is the set of real numbers. Moudafi [1], Moudafi and Thera [2], Peng and Yao [3,4], Takahashi and Takahashi [5] considered the following generalized equilibrium problem:

$$
\text { Find } x \in C \text { Such that } F(x, y)+\langle B x, y-x\rangle \geq 0, \forall y \in C \text {. }
$$

The set of solutions of (1.1) is denoted by $\operatorname{GEP}(F, B)$. If $B=0$, the generalized equilibrium problem (1.1) becomes the equilibrium problem for $F: C \times C \rightarrow R$, which is to find $x \in C$ such that

$$
F(x, y) \geq 0 \text { for all } y \in C .
$$

The set of solutions of (1.2) is denoted by $E P(F)$.

The problem (1.1) is very general in the sense that it includes, as special cases, optimization problems, variational inequalities, minimax problems, Nash equilibrium problem in noncooperative games, and others; see for instance [1-7].

Recall that a mapping $S: C \rightarrow C$ is nonexpansive if there holds that

$$
\|S x-S y\| \leq\|x-y\| \text { for all } x, y \in C \text {. }
$$

We denote the set of fixed points of $S$ by $\operatorname{Fix}(S)$.

\section{SpringerOpen ${ }^{\circ}$}

(c) 2011 Peng; licensee Springer. This is an Open Access article distributed under the terms of the Creative Commons Attribution License (http://creativecommons.org/licenses/by/2.0), which permits unrestricted use, distribution, and reproduction in any medium, provided the original work is properly cited. 
Let the mapping $A: C \rightarrow H$ be monotone and $k$-Lipschitz-continuous. The variational inequality problem is to find $x \in C$ such that

$$
\langle A x, y-x\rangle \geq 0
$$

for all $y \in C$. The set of solutions of the variational inequality problem is denoted by $V I(C, A)$.

Several algorithms have been proposed for finding the solution of problem (1.1). Moudafi [1] introduced an iterative scheme for finding a common element of the set of solutions of problem (1.1) and the set of fixed points of a nonexpansive mapping in a Hilbert space, and proved a weak convergence theorem. Moudafi and Thera [2] introduced an auxiliary scheme for finding a solution of problem (1.1) in a Hilbert space and obtained a weak convergence theorem. Peng and Yao [3,4] introduced some iterative schemes for finding a common element of the set of solutions of problem (1.1), the set of fixed points of a nonexpansive mapping and the set of solutions of the variational inequality for a monotone, Lipschitz-continuous mapping and obtain both strong convergence theorems, and weak convergence theorems for the sequences generated by the corresponding processes in Hilbert spaces. Takahashi and Takahashi [5] introduced an iterative scheme for finding a common element of the set of solutions of problem (1.1) and the set of fixed points of a nonexpansive mapping in a Hilbert space, and proved a strong convergence theorem.

Some methods also have been proposed to solve the problem (1.2); see, for instance, [8-19] and the references therein. Takahashi and Takahashi [9] introduced an iterative scheme by the viscosity approximation method for finding a common element of the set of solutions of problem (1.2) and the set of fixed points of a non-expansive mapping, and proved a strong convergence theorem in a Hilbert space. Su et al. [10] introduced and researched an iterative scheme by the viscosity approximation method for finding a common element of the set of solutions of problem (1.2) and the set of fixed points of a nonexpansive mapping and the set of solutions of the variational inequality problem for an $\alpha$-inverse-strongly monotone mapping in a Hilbert space. Tada and Takahashi [11] introduced two iterative schemes for finding a common element of the set of solutions of problem (1.2) and the set of fixed points of a nonexpansive mapping in a Hilbert space, and obtained both strong convergence and weak convergence theorems. Plubtieng and Punpaeng [12] introduced an iterative processes based on the extragradient method for finding the common element of the set of fixed points of a nonexpansive mapping, the set of solutions of an equilibrium problem and the set of solutions of variational inequality problem for an $\alpha$-inverse-strongly monotone mapping. Chang et al. [13] introduced an iterative processes based on the extragradient method for finding the common element of the set of solutions of an equilibrium problem, the set of common fixed point for a family of infinitely nonexpansive mappings and the set of solutions of variational inequality problem for an $\alpha$-inverse-strongly monotone mapping. Yao et al. [14] and Ceng and Yao [15] introduced some iterative viscosity approximation schemes for finding the common element of the set of solutions of problem (1.2) and the set of fixed points of a family of infinitely nonexpansive mappings in a Hilbert space. Colao et al. [16] introduced an iterative viscosity approximation scheme for finding a common element of the set of solutions of problem (1.2) 
and the set of fixed points of a family of finitely nonexpansive mappings in a Hilbert space. We observe that the algorithms in [13-16] involves the $W$-mapping generated by a family of infinitely (finitely) nonexpansive mappings which is an effective tool in nonlinear analysis (see $[20,21]$ ). However, the $W$-mapping generated by a family of infinitely (finitely) nonexpansive mappings is too completed to use for finding the common element of the set of solutions of problem (1.2) and the set of fixed points of a family of infinitely (finitely) nonexpansive mappings. It is natural to raise and to give an answer to the following question: Can one construct algorithms for finding a common element of the set of solutions of a generalized equilibrium problem (an equilibrium problem), the common set of fixed points of a family of infinitely nonexpansive mappings and the set of solutions of a variational inequality without the $W$-mapping generated by a family of infinitely (finitely) nonexpansive mappings? In this article, we will give a positive answer to this question.

Recently, OHaraa et al. [22] introduced and researched an iterative approach for finding a nearest point of infinitely many nonexpansive mappings in a Hilbert spaces without using the $\mathrm{W}$-mapping generated by a family of infinitely (finitely) nonexpansive mappings. Inspired by the ideas in [1-6,8-16,22] and the references therein, we introduce some new iterative schemes based on the extragradient method (and the hybrid method) for finding a common element of the set of solutions of a generalized equilibrium problem, the set of fixed points of a family of infinitely nonexpansive mappings, and the set of solutions of the variational inequality for a monotone, Lipschitz-continuous mapping without using the $W$-mapping generated by a family of infinitely (finitely) nonexpansive mappings. We obtain both strong convergence theorems and weak convergence theorems for the sequences generated by the corresponding processes. The results in this article generalize, improve, and unify some well-known convergence theorems in the literature.

\section{Preliminaries}

Let $H$ be a real Hilbert space with inner product $\langle\cdot, \cdot\rangle$ and norm $\|\cdot\|$. Let $C$ be a nonempty closed convex subset of $H$. Let symbols $\rightarrow$ and $\rightarrow$ denote strong and weak convergences, respectively. In a real Hilbert space $H$, it is well known that

$$
\|\lambda x+(1-\lambda) y\|^{2}=\lambda\|x\|^{2}+(1-\lambda)\|y\|^{2}-\lambda(1-\lambda)\|x-y\|^{2}
$$

for all $x, y \in H$ and $\lambda \in[0,1]$.

For any $x \in H$, there exists the unique nearest point in $C$, denoted by $P_{C}(x)$, such that $\left\|x-P_{C}(x)\right\| \leq\|x-y\|$ for all $y \in C$. The mapping $P_{C}$ is called the metric projection of $H$ onto $C$. We know that $P_{C}$ is a nonexpansive mapping from $H$ onto $C$. It is also known that $P_{C} x \in C$ and

$$
\left\langle x-P_{C}(x), P_{C}(x)-y\right\rangle \geq 0
$$

for all $x \in H$ and $y \in C$.

It is easy to see that (2.1) is equivalent to

$$
\|x-y\|^{2} \geq\left\|x-P_{C}(x)\right\|^{2}+\left\|y-P_{C}(x)\right\|^{2}
$$

for all $x \in H$ and $y \in C$. 
A mapping $A$ of $C$ into $H$ is called monotone if

$$
\langle A x-A y, x-y\rangle \geq 0
$$

for all $x, y \in C$. A mapping $A$ of $C$ into $H$ is called $\alpha$-inverse-strongly monotone if there exists a positive real number $\alpha$ such that

$$
\langle x-y, A x-A y\rangle \geq \alpha\|A x-A y\|^{2}
$$

for all $x, y \in C$. A mapping $A: C \rightarrow H$ is called $k$-Lipschitz-continuous if there exists a positive real number $k$ such that

$$
\|A x-A y\| \leq k\|x-y\|
$$

for all $x, y \in C$. It is easy to see that if $A$ is $\alpha$-inverse-strongly monotone, then $A$ is monotone and Lipschitz-continuous. The converse is not true in general. The class of $\alpha$-inverse-strongly monotone mappings does not contain some important classes of mappings even in a finite-dimensional case. For example, if the matrix in the corresponding linear complementarity problem is positively semidefinite, but not positively definite, then the mapping $A$ will be monotone and Lipschitz-continuous, but not $\alpha$ inverse-strongly monotone (see [23]).

Let $A$ be a monotone mapping of $C$ into $H$. In the context of the variational inequality problem, the characterization of projection (2.1) implies the following:

$$
u \in \operatorname{VI}(C, A) \Rightarrow u=P_{C}(u-\lambda A u), \lambda>0 .
$$

and

$$
u=P_{C}(u-\lambda A u) \text { for some } \lambda>0 \Rightarrow u \in \operatorname{VI}(C, A) .
$$

It is also known that $H$ satisfies the Opial's condition [24], i.e., for any sequence $\left\{x_{n}\right\}$ $\subset H$ with $x_{n} \rightarrow x$, the inequality

$$
\liminf _{n \rightarrow \infty}\left\|x_{n}-x\right\|<\liminf _{n \rightarrow \infty}\left\|x_{n}-y\right\|
$$

holds for every $y \in H$ with $x \neq y$.

A set-valued mapping $T: H \rightarrow 2^{H}$ is called monotone if for all $x, y \in H, f \in T x$ and $g \in$ $T y$ imply $\langle x-y, f-g\rangle \geq 0$. A monotone mapping $T: H \rightarrow 2^{H}$ is maximal if its graph $G(T)$ of $T$ is not properly contained in the graph of any other monotone mapping. It is known that a monotone mapping $T$ is maximal if and only if for $(x, f) \in H \times H,\langle x-y, f-g\rangle \geq 0$ for every $(y, g) \in G(T)$ implies $f \in T x$. Let A be a monotone, $k$-Lipschitz-continuous mapping of $C$ into $H$ and $N_{C} v$ be normal cone to $C$ at $v \in C$, i.e., $N_{C^{v}}=\{w \in H:\langle v-u$, $w\rangle \geq 0, \forall u \in C$. Define

$$
T v= \begin{cases}A v+N_{C} v & \text { if } v \in C, \\ \varnothing & \text { if } v \notin C .\end{cases}
$$

Then, $T$ is maximal monotone and $0 \in T v$ if and only if $v \in V I(C, A)$ (see [25]).

For solving the equilibrium problem, let us assume that the bifunction $F$ satisfies the following condition:

(A1) $F(x, x)=0$ for all $x \in C$;

(A2) $F$ is monotone, i.e., $F(x, y)+F(y, x) \leq 0$ for any $x, y \in C$; 
(A3) for each $x, y, z \in C$,

$$
\lim _{t \downarrow 0} F(t z+(1-t) x, y) \leq F(x, y)
$$

(A4) for each $x \in C, y \mapsto F(x, y)$ is convex and lower semicontinuous.

We recall some lemmas which will be needed in the rest of this article.

Lemma 2.1.[7] Let $C$ be a nonempty closed convex subset of $H$, let $F$ be a bifunction from $C \times C$ to $R$ satisfying (A1)-(A4). Let $r>0$ and $x \in H$. Then, there exists $z \in C$ such that

$$
F(z, y)+\frac{1}{r}\langle y-z, z-x\rangle \geq 0, \quad \text { for all } y \in C .
$$

Lemma 2.2.[8] Let $C$ be a nonempty closed convex subset of $H$, let $F$ be a bi-function from $C \times C$ to $R$ satisfying (A1)-(A4). For $r>0$ and $x \in H$, define a mapping $T r$ : $H \rightarrow C$ as follows:

$$
T_{r}(x)=\left\{z \in C: F(z, y)+\frac{1}{r}\langle y-z, z-x\rangle \geq 0, \forall y \in C\right\}
$$

for all $x \in H$. Then, the following statements hold:

(1) $T_{r}$ is single-valued;

(2) $T_{r}$ is firmly nonexpansive, i.e., for any $x, y \in H$,

$$
\left\|T_{r}(x)-T_{r}(y)\right\|^{2} \leq\left\langle T_{r}(x)-T_{r}(y), x-y\right\rangle ;
$$

(3) $F\left(T_{r}\right)=E P(F)$;

(4) $E P(F)$ is closed and convex.

\section{The main results}

We first show a strong convergence of an iterative algorithm based on extragradient and hybrid methods which solves the problem of finding a common element of the set of solutions of a generalized equilibrium problem, the set of fixed points of a family of infinitely nonexpansive mappings, and the set of solutions of the variational inequality for a monotone, Lipschitz-continuous mapping in a Hilbert space.

Theorem 3.1. Let $C$ be a nonempty closed convex subset of a real Hilbert space $H$. Let $F$ be a bifunction from $C \times C$ to $R$ satisfying (A1)-(A4). Let $A$ be a monotone and $k$-Lipschitz-continuous mapping of $C$ into $H$ and $B$ be an $\alpha$-inverse-strongly monotone mapping of $C$ into $H$. Let $S_{1}, S_{2}, \ldots$ be a family of infinitely nonexpansive mappings of $C$ into itself such that $\Omega=\cap_{i=1}^{\infty} \operatorname{Fix}\left(S_{i}\right) \cap \operatorname{VI}(C, A) \cap \operatorname{GEP}(F, B) \neq \emptyset$. Assume that for all $i \in$ $\{1,2, \ldots\}$ and for any bounded subset $K$ of $C$, thenthere holds

$$
\lim _{n \rightarrow \infty} \sup _{x \in K}\left\|S_{n} x-S_{i}\left(S_{n} x\right)\right\|=0 .
$$

Let $\left\{x_{n}\right\},\left\{u_{n}\right\},\left\{y_{n}\right\}$ and $\left\{z_{n}\right\}$ be sequences generated by

$$
\left\{\begin{array}{l}
x_{1}=x \in C, \\
F\left(u_{n}, y\right)+\left\langle B x_{n}, y-u_{n}\right\rangle+\frac{1}{r_{n}}\left\langle y-u_{n}, u_{n}-x_{n}\right\rangle \geq 0, \quad \forall y \in C, \\
y_{n}=\left(1-\gamma_{n}\right) u_{n}+\gamma_{n} P_{C}\left(u_{n}-\lambda_{n} A u_{n}\right), \\
z_{n}=\left(1-\alpha_{n}-\beta_{n}\right) x_{n}+\alpha_{n} y_{n}+\beta_{n} S_{n} P_{C}\left(u_{n}-\lambda_{n} A y_{n}\right), \\
C_{n}=\left\{z \in C:\left\|z_{n}-z\right\|^{2} \leq\left\|x_{n}-z\right\|^{2}+\left(3-3 \gamma_{n}+\alpha_{n}\right) b^{2}\left\|A u_{n}\right\|^{2}\right\}, \\
Q_{n}=\left\{z \in C:\left\langle x_{n}-z, x-x_{n}\right\rangle \geq 0\right\}, \\
x_{n+1}=P_{C_{n}} \cap Q_{n} x
\end{array}\right.
$$


for every $n=1,2, \ldots$ where $\left\{\lambda_{n}\right\} \subset[a, b]$ for some $a, b \in\left(0, \frac{1}{4 k}\right),\left\{r_{n}\right\} \subset[d, e]$ for some $d, e \in(0,2 \alpha)$, and $\left\{\alpha_{n}\right\},\left\{\beta_{n}\right\},\left\{\gamma_{n}\right\}$ are three sequences in $[0,1]$ satisfying the conditions:

(i) $\alpha_{n}+\beta_{n} \leq 1$ for all $n \in N$;

(ii) $\lim _{n \rightarrow \infty} \alpha_{n}=0$;

(iii) $\liminf _{n \rightarrow \infty} \beta_{n}>0$;

(iv) $\lim _{n \rightarrow \infty} \gamma_{n}=1$ and $\gamma_{n}>\frac{3}{4}$ for all $n \in N$;

Then, $\left\{x_{n}\right\},\left\{u_{n}\right\},\left\{y_{n}\right\}$ and $\left\{z_{n}\right\}$ converge strongly to $w=P_{\Omega}(x)$.

Proof. It is obvious that $C_{n}$ is closed, and $Q_{n}$ is closed and convex for every $n=1$, $2, \ldots$. Since

$$
C_{n}=\left\{z \in H:\left\|z_{n}-x_{n}\right\|^{2}+2\left\langle z_{n}-x_{n}, x_{n}-z\right\rangle \leq\left(3-3 \gamma_{n}+\alpha_{n}\right) b^{2}\left\|A u_{n}\right\|^{2}\right\},
$$

we also have that $C_{n}$ is convex for every $n=1,2, \ldots$ It is easy to see that $\left\langle x_{n}-z, x-\right.$ $\left.x_{n}\right\rangle \geq 0$ for all $z \in Q_{n}$ and by (2.1), $x_{n}=P_{Q_{n}} x$. Let $t_{n}=P_{C}\left(u_{n}-\lambda_{n} A y_{n}\right)$ for every $n=1$, $2, \ldots$. Let $u \in \Omega$ and let $\left\{T_{r_{n}}\right\}>$ be a sequence of mappings defined as in Lemma 2.2. Then $u=P_{C}\left(u-\lambda_{n} A u\right)=T_{r_{n}}\left(u-r_{n} B u\right)$. From $u_{n}=T_{r_{n}}\left(x_{n}-r_{n} B x_{n}\right) \in C$ and the $\alpha-$ inverse strongly monotonicity of $B$, we have

$$
\begin{gathered}
\left\|u_{n}-u\right\|^{2}=\left\|T_{r_{n}}\left(x_{n}-r_{n} B x_{n}\right)-T_{r_{n}}\left(u-r_{n} B u\right)\right\|^{2} \\
\leq\left\|x_{n}-r_{n} B x_{n}-\left(u-r_{n} B u\right)\right\|^{2} \\
\leq\left\|x_{n}-u\right\|^{2}-2 r_{n}\left\langle x_{n}-u, B x_{n}-B u\right\rangle+r_{n}^{2}\left\|B x_{n}-B u\right\|^{2} \\
\leq\left\|x_{n}-u\right\|^{2}-2 r_{n} \alpha\left\|B x_{n}-B u\right\|^{2}+r_{n}^{2}\left\|B x_{n}-B u\right\|^{2} \\
=\left\|x_{n}-u\right\|^{2}+r_{n}\left(r_{n}-2 \alpha\right)\left\|B x_{n}-B u\right\|^{2} \\
\leq\left\|x_{n}-u\right\|^{2} .
\end{gathered}
$$

From (2.2), the monotonicity of $A$, and $u \in V I(C, A)$, we have

$$
\begin{aligned}
& \left\|t_{n}-u\right\|^{2} \leq\left\|u_{n}-\lambda_{n} A y_{n}-u\right\|^{2}-\left\|u_{n}-\lambda_{n} A y_{n}-t_{n}\right\|^{2} \\
& =\left\|u_{n}-u\right\|^{2}-\left\|u_{n}-t_{n}\right\|^{2}+2 \lambda_{n}\left\langle A y_{n}, u-t_{n}\right\rangle \\
& =\left\|u_{n}-u\right\|^{2}-\left\|u_{n}-t_{n}\right\|^{2}+2 \lambda_{n}\left(\left\langle A y_{n}-A u, u-y_{n}\right\rangle+\left\langle A u, u-y_{n}\right\rangle+\left\langle A y_{n}, y_{n}-t_{n}\right\rangle\right) \\
& \quad \leq\left\|u_{n}-u\right\|^{2}-\left\|u_{n}-t_{n}\right\|^{2}+2 \lambda_{n}\left\langle A y_{n}, y_{n}-t_{n}\right\rangle \\
& \leq\left\|u_{n}-u\right\|^{2}-\left\|u_{n}-y_{n}\right\|^{2}-2\left\langle u_{n}-y_{n}, y_{n}-t_{n}\right\rangle-\left\|y_{n}-t_{n}\right\|^{2}+2 \lambda_{n}\left\langle A y_{n}, y_{n}-t_{n}\right\rangle \\
& =\left\|u_{n}-u\right\|^{2}-\left\|u_{n}-y_{n}\right\|^{2}-\left\|y_{n}-t_{n}\right\|^{2}+2\left\langle u_{n}-\lambda_{n} A y_{n}-y_{n}, t_{n}-y_{n}\right\rangle .
\end{aligned}
$$

Further, Since $y_{n}=\left(1-\gamma_{n}\right) u_{n}+\gamma_{n} P_{C}\left(u_{n}-\lambda_{n} A u_{n}\right)$ and $A$ is $k$-Lipschitz-continuous, we have

$$
\begin{aligned}
& \left\langle u_{n}-\lambda_{n} A y_{n}-\gamma_{n}, t_{n}-\gamma_{n}\right\rangle \\
= & \left\langle u_{n}-\lambda_{n} A u_{n}-\gamma_{n}, t_{n}-y_{n}\right\rangle+\left\langle\lambda_{n} A u_{n}-\lambda_{n} A y_{n}, t_{n}-\gamma_{n}\right\rangle \\
\leq & \left\langle u_{n}-\lambda_{n} A u_{n}-\left(1-\gamma_{n}\right) u_{n}-\gamma_{n} P_{C}\left(u_{n}-\lambda_{n} A u_{n}\right), t_{n}-y_{n}\right\rangle+\lambda_{n}\left\|A u_{n}-A y_{n}\right\|\left\|t_{n}-y_{n}\right\| \\
\leq & \gamma_{n}\left\langle u_{n}-\lambda_{n} A u_{n}-P_{C}\left(u_{n}-\lambda_{n} A u_{n}\right), t_{n}-\gamma_{n}\right\rangle-\left(1-\gamma_{n}\right) \lambda_{n}\left\langle A u_{n}, t_{n}-\gamma_{n}\right\rangle+\lambda_{n} k\left\|u_{n}-\gamma_{n}\right\|\left\|t_{n}-y_{n}\right\| .
\end{aligned}
$$


In addition, from the definition of $P_{C}$, we have

$$
\begin{aligned}
& \left\langle u_{n}-\lambda_{n} A u_{n}-P_{C}\left(u_{n}-\lambda_{n} A u_{n}\right), t_{n}-\gamma_{n}\right\rangle \\
= & \left\langle u_{n}-\lambda_{n} A u_{n}-P_{C}\left(u_{n}-\lambda_{n} A u_{n}\right), t_{n}-\left(1-\gamma_{n}\right) u_{n}-\gamma_{n} P_{C}\left(u_{n}-\lambda_{n} A u_{n}\right)\right\rangle \\
= & \left(1-\gamma_{n}\right)\left\langle u_{n}-\lambda_{n} A u_{n}-P_{C}\left(u_{n}-\lambda_{n} A u_{n}\right), t_{n}-u_{n}\right\rangle \\
+ & \gamma_{n}\left\langle u_{n}-\lambda_{n} A u_{n}-P_{C}\left(u_{n}-\lambda_{n} A u_{n}\right), t_{n}-P_{C}\left(u_{n}-\lambda_{n} A u_{n}\right)\right\rangle \\
& \leq\left(1-\gamma_{n}\right)\left\|u_{n}-\lambda_{n} A u_{n}-P_{C}\left(u_{n}-\lambda_{n} A u_{n}\right)\right\|\left\|t_{n}-u_{n}\right\| \\
\leq & \left(1-\gamma_{n}\right) \lambda_{n}\left\|u_{n}-A u_{n}-u_{n}\right\|\left(\left\|t_{n}-\gamma_{n}\right\|+\left\|y_{n}-u_{n}\right\|\right) \\
\leq & \left(1-\gamma_{n}\right) \lambda_{n}\left\|A u_{n}\right\|\left(\left\|t_{n}-\gamma_{n}\right\|+\left\|y_{n}-u_{n}\right\|\right) .
\end{aligned}
$$

It follows from $b<\frac{1}{4 k}, \gamma_{n}>\frac{3}{4}$ and (3.2) that

$$
\begin{gathered}
\left\|t_{n}-u\right\|^{2} \leq\left\|u_{n}-u\right\|^{2}-\left\|u_{n}-y_{n}\right\|^{2}-\left\|y_{n}-t_{n}\right\|^{2}+2 \gamma_{n}\left(1-\gamma_{n}\right) b\left\|A u_{n}\right\|\left(\left\|t_{n}-\gamma_{n}\right\|+\left\|y_{n}-u_{n}\right\|\right) \\
+2\left(1-\gamma_{n}\right) b\left\|A u_{n}\right\|\left\|t_{n}-\gamma_{n}\right\|+2 b k\left\|u_{n}-\gamma_{n}\right\|\left\|t_{n}-y_{n}\right\| \\
\leq\left\|u_{n}-u\right\|^{2}-\left\|u_{n}-\gamma_{n}\right\|^{2}-\left\|y_{n}-t_{n}\right\|^{2}+\left(1-\gamma_{n}\right)\left(2 b^{2}\left\|A u_{n}\right\|^{2}+\left\|t_{n}-y_{n}\right\|^{2}+\left\|y_{n}-u_{n}\right\|^{2}\right) \\
+\left(1-\gamma_{n}\right)\left(b^{2}\left\|A u_{n}\right\|^{2}+\left\|t_{n}-\gamma_{n}\right\|^{2}\right)+b k\left(\left\|u_{n}-\gamma_{n}\right\|^{2}+\left\|t_{n}-\gamma_{n}\right\|^{2}\right) \\
=\left\|u_{n}-u\right\|^{2}-\left(\gamma_{n}-b k\right)\left\|u_{n}-\gamma_{n}\right\|^{2}+\left(1-2 \gamma_{n}+b k\right)\left\|t_{n}-\gamma_{n}\right\|^{2}+3\left(1-\gamma_{n}\right) b^{2}\left\|A u_{n}\right\|^{2} \\
\leq\left\|u_{n}-u\right\|^{2}+3\left(1-\gamma_{n}\right) b^{2}\left\|A u_{n}\right\|^{2} \\
\leq\left\|x_{n}-u\right\|^{2}+3\left(1-\gamma_{n}\right) b^{2}\left\|A u_{n}\right\|^{2} .
\end{gathered}
$$

In addition, from $u \in V I(C, A)$ and (3.2), we have

$$
\begin{gathered}
\left\|y_{n}-u\right\|^{2}=\left\|\left(1-\gamma_{n}\right)\left(u_{n}-u\right)+\gamma_{n}\left(P_{C}\left(u_{n}-\lambda_{n} A u_{n}\right)-u\right)\right\|^{2} \\
\leq\left(1-\gamma_{n}\right)\left\|u_{n}-u\right\|^{2}+\gamma_{n}\left\|P_{C}\left(u_{n}-\lambda_{n} A u_{n}\right)-P_{C}(u)\right\|^{2} \\
\leq\left(1-\gamma_{n}\right)\left\|u_{n}-u\right\|^{2}+\gamma_{n}\left\|u_{n}-\lambda_{n} A u_{n}-u\right\|^{2} \\
\leq\left(1-\gamma_{n}\right)\left\|u_{n}-u\right\|^{2}+\gamma_{n}\left[\left\|u_{n}-u\right\|^{2}-2 \lambda_{n}\left\langle A u_{n}, u_{n}-u\right\rangle+\lambda_{n}^{2}\left\|A u_{n}\right\|^{2}\right] \\
\leq\left\|u_{n}-u\right\|^{2}+b^{2}\left\|A u_{n}\right\|^{2} \\
\leq\left\|x_{n}-u\right\|^{2}+b^{2}\left\|A u_{n}\right\|^{2} .
\end{gathered}
$$

Therefore, from (3.2) to (3.4) and $z_{n}=\left(1-\alpha_{n}-\beta_{n}\right) x_{n}+\alpha_{n} y_{n}+\beta_{n} S_{n} t_{n}$ and $u=S_{n} u$, we have

$$
\begin{gathered}
\left\|z_{n}-u\right\|^{2}=\left\|\left(1-\alpha_{n}-\beta_{n}\right) x_{n}+\alpha_{n} y_{n}+\beta_{n} S_{n} t_{n}-u\right\|^{2} \\
\leq\left(1-\alpha_{n}-\beta_{n}\right)\left\|x_{n}-u\right\|^{2}+\alpha_{n}\left\|y_{n}-u\right\|^{2}+\beta_{n}\left\|S_{n} t_{n}-u\right\|^{2} \\
\leq\left(1-\alpha_{n}-\beta_{n}\right)\left\|x_{n}-u\right\|^{2}+\alpha_{n}\left\|y_{n}-u\right\|^{2}+\beta_{n}\left\|t_{n}-u\right\|^{2} \\
\leq\left(1-\alpha_{n}-\beta_{n}\right)\left\|x_{n}-u\right\|^{2}+\alpha_{n}\left[\left\|u_{n}-u\right\|^{2}+b^{2}\left\|A u_{n}\right\|^{2}\right] \\
+\beta_{n}\left[\left\|u_{n}-u\right\|^{2}+3\left(1-\gamma_{n}\right) b^{2}\left\|A u_{n}\right\|^{2}\right] \\
\left.\leq\left\|x_{n}-u\right\|^{2}+\left(3-3 \gamma_{n}+\alpha_{n}\right) b^{2}\left\|A u_{n}\right\|^{2}\right],
\end{gathered}
$$

for every $n=1,2, \ldots$ and hence $u \in C_{n}$. So, $\Omega \subset C_{n}$ for every $n=1,2, \ldots$. Next, let us show by mathematical induction that $x_{n}$ is well defined and $\Omega \subset C_{n} \cap Q_{n}$ for every $n$ $=1,2, \ldots$. For $n=1$ we have $x_{1}=x \in C$ and $Q_{1}=C$. Hence, we obtain $\Omega \subset C_{1} \cap Q_{1}$. Suppose that $x_{k}$ is given and $\Omega \subset C_{k} \cap Q_{k}$ for some $k \in N$. Since $\Omega$ is nonempty, $C_{k} \cap$ $Q_{k}$ is a nonempty closed convex subset of $H$. Hence, there exists a unique element $x_{k+1}$ $\in C_{k} \cap Q_{k}$ such that $x_{k+1}=P_{C_{k} \cap Q_{k}} x$. It is also obvious that there holds $\left\langle x_{k+1}-z, x-x_{k}\right.$ $\left.{ }_{+1}\right\rangle \geq 0$ for every $z \in C_{k} \cap Q_{k}$. Since $\Omega \subset C_{k} \cap Q_{k}$, we have $\left\langle x_{k+1}-z, x-x_{k+1}\right\rangle \geq 0$ for every $z \in \Omega$ and hence $\Omega \subset Q_{k+1}$. Therefore, we obtain $\Omega \subset C_{k+1} \cap Q_{k+1}$. 
Let $l_{0}=P_{\Omega^{2}}$. From $x_{n+1}=P_{C_{n} \cap Q_{n}} x$ and $l_{0} \mathrm{v} \Omega \subset C_{n} \cap Q_{n}$, we have

$$
\left\|x_{n+1}-x\right\| \leq\left\|l_{0}-x\right\|
$$

for every $n=1,2, \ldots$. Therefore, $\left\{x_{n}\right\}$ is bounded. From (3.2) to (3.5) and the lipschitz continuity of $A$, we also obtain that $\left\{u_{n}\right\},\left\{y_{n}\right\},\left\{A u_{n}\right\},\left\{t_{n}\right\}$ and $\left\{z_{n}\right\}$ are bounded. Since $x_{n+1} \in C_{n} \cap Q_{n} \subset C_{n}$ and $x_{n}=P_{Q_{n}} x$, we have

$$
\left\|x_{n}-x\right\| \leq\left\|x_{n+1}-x\right\|
$$

for every $n=1,2, \ldots$. It follows from (3.6) that $\lim _{n \rightarrow \infty}\left\|x_{n}-x\right\|$ exists.

Since $x_{n}=P_{Q_{n}} x$ and $x_{n+1} \in Q_{n}$, using (2.2), we have

$$
\left\|x_{n+1}-x_{n}\right\|^{2} \leq\left\|x_{n+1}-x\right\|^{2}-\left\|x_{n}-x\right\|^{2}
$$

for every $n=1,2, \ldots$. This implies that

$$
\lim _{n \rightarrow \infty}\left\|x_{n+1}-x_{n}\right\|=0 .
$$

Since $x_{n+1} \in C_{n}$, we have $\left\|z_{n}-x_{n+1}\right\|^{2} \leq\left\|x_{n}-x_{n+1}\right\|^{2}+\left(3-3 \gamma_{n}+\alpha_{n}\right) b^{2}\left\|A u_{n}\right\|^{2}$ and hence it follows from $\lim _{n \rightarrow \infty} \gamma_{n}=1$ and $\lim _{n \rightarrow \infty} \alpha_{n}=0$ that $\lim _{n \rightarrow \infty}|| z_{n}-x_{n+1}||=0$. Since

$$
\left\|x_{n}-z_{n}\right\| \leq\left\|x_{n}-x_{n+1}||+|| x_{n+1}-z_{n}\right\|
$$

for every $n=1,2, \ldots$, we have $\left\|x_{n}-z_{n}\right\| \rightarrow 0$.

For $u \in \Omega$, from (3.5), we obtain

$$
\begin{gathered}
\left\|z_{n}-u\right\|^{2}-\left\|x_{n}-u\right\|^{2} \\
\leq\left(-\alpha_{n}-\beta_{n}\right)\left\|x_{n}-u\right\|^{2}+\alpha_{n}\left\|\gamma_{n}-u\right\|^{2}+\beta_{n}\left\|S_{n} t_{n}-u\right\|^{2} \\
\leq\left(3-3 \gamma_{n}+\alpha_{n}\right) b^{2}\left\|A u_{n}\right\|^{2} .
\end{gathered}
$$

Since $\lim _{n \rightarrow \infty} \gamma_{n}=1$ and $\lim _{n \rightarrow \infty} \alpha_{n}=0,\left\{x_{n}\right\},\left\{y_{n}\right\},\left\{A u_{n}\right\}$, and $\left\{z_{n}\right\}$ are bounded, we have

$$
\lim _{n \rightarrow \infty} \beta_{n}\left(\left\|S_{n} t_{n}-u\right\|^{2}-\left\|x_{n}-u\right\|^{2}\right)=0 .
$$

By $\lim \inf _{n \rightarrow \infty} \beta_{n}>0$, we get

$$
\lim _{n \rightarrow \infty}\left\|S_{n} t_{n}-u\right\|^{2}-\left\|x_{n}-u\right\|^{2}=0 .
$$

From (3.3) and $u=S_{n} u$, we have

$$
\begin{gathered}
\lim _{n \rightarrow \infty}\left\|S_{n} t_{n}-u\right\|^{2}-\left\|x_{n}-u\right\|^{2} \leq \lim _{n \rightarrow \infty}\left\|t_{n}-u\right\|^{2}-\left\|x_{n}-u\right\|^{2} \\
\leq \lim _{n \rightarrow \infty} 3\left(1-\gamma_{n}\right) b^{2}\left\|A u_{n}\right\|^{2}=0 .
\end{gathered}
$$

Thus, $\lim _{n \rightarrow \infty}\left\|t_{n}-u\right\|^{2}-\left\|x_{n}-u\right\|^{2}=0$.

From (3.3) and (3.2), we have

$$
\begin{aligned}
& \left(\gamma_{n}-b k\right)\left\|u_{n}-y_{n}\right\|^{2}+\left(2 \gamma_{n}-1-b k\right)\left\|t_{n}-\gamma_{n}\right\|^{2} \\
& \leq\left\|x_{n}-u\right\|^{2}-\left\|t_{n}-u\right\|^{2}+3\left(1-\gamma_{n}\right) b^{2}\left\|A u_{n}\right\|^{2} .
\end{aligned}
$$

It follows that

$$
\lim _{n \rightarrow \infty}\left(\gamma_{n}-b k\right)\left\|u_{n}-\gamma_{n}\right\|^{2}+\left(2 \gamma_{n}-1-b k\right)\left\|t_{n}-\gamma_{n}\right\|^{2}=0 .
$$


The assumptions on $\gamma_{n}$ and $\lambda_{n}$ imply that $\gamma_{n}-b k>\frac{1}{2}$ and $2 \gamma_{n}-1-b k>\frac{1}{4}$. Consequently, $\lim _{n \rightarrow \infty}|| u_{n}-y_{n}\left\|=\lim _{n \rightarrow \infty}\right\| t_{n}-y_{n} \|=0$. Since $A$ is Lipschitz-continuous, we have $\lim _{n \rightarrow \infty}|| A t_{n}-A y_{n} \|=0$. It follows from $\left\|u_{n}-t_{n}\right\| \leq\left\|u_{n}-y_{n}\right\|+\left\|t_{n}-y_{n}\right\|$ that $\lim _{n \rightarrow \infty}\left\|u_{n}-t_{n}\right\|=0$.

We rewrite the definition of $z_{n}$ as

$$
z_{n}-x_{n}=\alpha_{n}\left(y_{n}-x_{n}\right)+\beta_{n}\left(S_{n} t_{n}-x_{n}\right) .
$$

From $\lim _{n \rightarrow \infty}|| z_{n}-x_{n} \|=0, \lim _{n \rightarrow \infty} \alpha_{n}=0$, the boundedness of $\left\{x_{n}\right\},\left\{y_{n}\right\}$ and lim $\inf _{n \rightarrow \infty} \beta_{n}>0$ we infer that $\lim _{n \rightarrow \infty}|| S_{n} t_{n}-x_{n} \|=0$.

By (3.2)-(3.5), we have

$$
\begin{aligned}
& \left\|z_{n}-u\right\|^{2} \leq\left(1-\alpha_{n}-\beta_{n}\right)\left\|x_{n}-u\right\|^{2}+\alpha_{n}\left[\left\|u_{n}-u\right\|^{2}+b^{2}\left\|A u_{n}\right\|^{2}\right]+\beta_{n}\left[\left\|u_{n}-u\right\|^{2}+3\left(1-\gamma_{n}\right) b^{2}\left\|A u_{n}\right\|^{2}\right] \\
& \leq\left(1-\alpha_{n}-\beta_{n}\right)\left\|x_{n}-u\right\|^{2}+\alpha_{n}\left[\left\|x_{n}-u\right\|^{2}+r_{n}\left(r_{n}-2 \alpha\right)\left\|B x_{n}-B u\right\|^{2}+b^{2}\left\|A u_{n}\right\|^{2}\right] \\
& \quad+\beta_{n}\left[\left\|x_{n}-u\right\|^{2}+r_{n}\left(r_{n}-2 \alpha\right)\left\|B x_{n}-B u\right\|^{2}+3\left(1-\gamma_{n}\right) b^{2}\left\|A u_{n}\right\|^{2}\right] \\
& \left.\leq\left\|x_{n}-u\right\|^{2}+\left(\alpha_{n}+\beta_{n}\right) r_{n}\left(r_{n}-2 \alpha\right)\left\|B x_{n}-B u\right\|^{2}+\left(3 \beta_{n}-3 \beta_{n} \gamma_{n}+\alpha_{n}\right) b^{2}\left\|A u_{n}\right\|^{2}\right] .
\end{aligned}
$$

Hence, we have

$$
\begin{gathered}
\left(\alpha_{n}+\beta_{n}\right) d(2 \alpha-e)\left\|B x_{n}-B u\right\|^{2} \\
\leq\left(\alpha_{n}+\beta_{n}\right) r_{n}\left(2 \alpha-r_{n}\right)\left\|B x_{n}-B u\right\|^{2} \\
\leq\left\|x_{n}-u\right\|^{2}-\left\|z_{n}-u\right\|^{2}+\left(3 \beta_{n}-3 \beta_{n} \gamma_{n}+\alpha_{n}\right) b^{2}\left\|A u_{n}\right\|^{2} \\
\leq\left(\left\|x_{n}-u\right\|+\left\|z_{n}-u\right\|\right)\left\|x_{n}-z_{n}\right\|+\left(3 \beta_{n}-3 \beta_{n} \gamma_{n}+\alpha_{n}\right) b^{2}\left\|A u_{n}\right\|^{2} .
\end{gathered}
$$

Since $\lim _{n \rightarrow \infty} \alpha_{n}=1, \lim _{\inf _{n \rightarrow \infty}} \beta_{n}>0, \lim _{n \rightarrow \infty} \gamma_{n}=1,|| x_{n}-z_{n} \| \rightarrow 0$ and the sequences $\left\{x_{n}\right\}$ and $\left\{z_{n}\right\}$ are bounded, we obtain $\left\|B x_{n}-B_{u}\right\| \rightarrow 0$.

For $u \in \Omega$, we have, from Lemma 2.2,

$$
\begin{gathered}
\left\|u_{n}-u\right\|^{2}=\left\|T_{r_{n}}\left(x_{n}-r_{n} B x_{n}\right)-T_{r_{n}}\left(u-r_{n} B u\right)\right\|^{2} \\
\leq\left\langle T_{r_{n}}\left(x_{n}-r_{n} B x_{n}\right)-T_{r_{n}}\left(u-r_{n} B u\right), x_{n}-r_{n} B x_{n}-\left(u-r_{n} B u\right)\right\rangle \\
=\frac{1}{2}\left\{\left\|u_{n}-u\right\|^{2}+\left\|x_{n}-r_{n} B x_{n}-\left(u-r_{n} B u\right)\right\|^{2}-\left\|x_{n}-r_{n} B x_{n}-\left(u-r_{n} B u\right)-\left(u_{n}-u\right)\right\|^{2}\right\} \\
\leq \frac{1}{2}\left\{\left\|u_{n}-u\right\|^{2}+\left\|x_{n}-u\right\|^{2}-\left\|x_{n}-r_{n} B x_{n}-\left(u-r_{n} B u\right)-\left(u_{n}-u\right)\right\|^{2}\right\} \\
=\frac{1}{2}\left\{\left\|u_{n}-u\right\|^{2}+\left\|x_{n}-u\right\|^{2}-\left\|x_{n}-u_{n}\right\|^{2}+2 r_{n}\left\langle B x_{n}-B u, x_{n}-u_{n}\right\rangle-r_{n}^{2}\left\|B x_{n}-B u\right\|^{2}\right\} .
\end{gathered}
$$

Hence,

$$
\begin{aligned}
\left\|u_{n}-u\right\|^{2} \leq & \left\|x_{n}-u\right\|^{2}-\left\|x_{n}-u_{n}\right\|^{2}+2 r_{n}\left\langle B x_{n}-B u, x_{n}-u_{n}\right\rangle-r_{n}^{2}\left\|B x_{n}-B u\right\|^{2} \\
& \leq\left\|x_{n}-u\right\|^{2}-\left\|x_{n}-u_{n}\right\|^{2}+2 r_{n}\left\langle B x_{n}-B u, x_{n}-u_{n}\right\rangle .
\end{aligned}
$$

Then, by (3.5), we have

$$
\begin{aligned}
& \left\|z_{n}-u\right\|^{2} \leq\left(1-\alpha_{n}-\beta_{n}\right)\left\|x_{n}-u\right\|^{2}+\alpha_{n}\left[\left\|u_{n}-u\right\|^{2}+b^{2}\left\|A u_{n}\right\|^{2}\right]+\beta_{n}\left[\left\|u_{n}-u\right\|^{2}+3\left(1-\gamma_{n}\right) b^{2}\left\|A u_{n}\right\|^{2}\right] \\
& \leq\left(1-\alpha_{n}-\beta_{n}\right)\left\|x_{n}-u\right\|^{2}+\alpha_{n}\left[\left(\left\|x_{n}-u\right\|^{2}-\left\|x_{n}-u_{n}\right\|^{2}+2 r_{n}\left\langle B x_{n}-B u, x_{n}-u_{n}\right\rangle\right)+b^{2}\left\|A u_{n}\right\|^{2}\right] \\
& \quad+\beta_{n}\left[\left(\left\|x_{n}-u\right\|^{2}-\left\|x_{n}-u_{n}\right\|^{2}+2 r_{n}\left\langle B x_{n}-B u, x_{n}-u_{n}\right\rangle\right)+3\left(1-\gamma_{n}\right) b^{2}\left\|A u_{n}\right\|^{2}\right] \\
& \leq\left\|x_{n}-u\right\|^{2}+\left(-\alpha_{n}-\beta_{n}\right)\left\|x_{n}-u_{n}\right\|^{2}+2 r_{n}\left(\alpha_{n}+\beta_{n}\right)\left\|B x_{n}-B u\right\|\left\|x_{n}-u_{n}\right\|+\left(3 \beta_{n}-3 \beta_{n} \gamma_{n}+\alpha_{n}\right) b^{2}\left\|A u_{n}\right\|^{2}
\end{aligned}
$$

Hence,

$$
\begin{aligned}
& \left(\alpha_{n}+\beta_{n}\right)|| x_{n}-u_{n}\left\|^{2} \leq\right\| x_{n}-u\left\|^{2}-\right\| z_{n}-u\left\|^{2}+2 r_{n}\left(\alpha_{n}+\beta_{n}\right)\right\| B x_{n}-B u\|\| x_{n}-u_{n}\left\|+\left(3 \beta_{n}-3 \beta_{n} \gamma_{n}+\alpha_{n}\right) b^{2}\right\| A u_{n} \|^{2} \\
& \leq\left(\left\|x_{n}-u\right\|+\left\|z_{n}-u\right\|\right)\left\|x_{n}-z_{n}\right\|+2 r_{n}\left(\alpha_{n}+\beta_{n}\right)\left\|B x_{n}-B u\right\|\|\mid\| x_{n}-u_{n}\left\|+\left(3 \beta_{n}-3 \beta_{n} \gamma_{n}+\alpha_{n}\right) b^{2}\right\| A u_{n} \|^{2} .
\end{aligned}
$$

Since $\lim _{n \rightarrow \infty} \alpha_{n}=0, \liminf _{n \rightarrow \infty} \beta_{n}>0, \lim _{n \rightarrow \infty} \gamma_{n}=1,\left\|x_{n}-z_{n}\right\| \rightarrow 0,\left\|B x_{n}-B u\right\| \rightarrow 0$ and the sequences $\left\{x_{n}\right\},\left\{u_{n}\right\}$ and $\left\{z_{n}\right\}$ are bounded, we obtain $\left\|x_{n}-u_{n}\right\| \rightarrow 0$. From $\| z_{n}$ $t_{n} \| \leq|| z_{n}-x_{n}||+|| x_{n}-u_{n}||+|| u_{n}-t_{n}||$, we have $\left\|z_{n}-t_{n}\right\| \rightarrow 0$. 
From $\left\|t_{n}-x_{n}\right\| \leq\left\|t_{n}-u_{n}\right\|+|| x_{n}-u_{n} \|$, we also have $\left\|t_{n}-x_{n}\right\| \rightarrow 0$.

Since $z_{n}=\left(1-\alpha_{n}-\beta_{n}\right) x_{n}+\alpha_{n} y_{n}+\beta_{n} S_{n} t_{n}$, we have $\beta_{n}\left(S_{n} t_{n}-t_{n}\right)=\left(1-\alpha_{n}-\beta_{n}\right)\left(t_{n}-\right.$ $\left.x_{n}\right)+\alpha_{n}\left(t_{n}-y_{n}\right)+\left(z_{n}-t_{n}\right)$. Then

$$
\beta_{n}\left\|S_{n} t_{n}-t_{n}\right\| \leq\left(1-\alpha_{n}-\beta_{n}\right)\left\|t_{n}-x_{n}\right\|+\alpha_{n}\left\|t_{n}-y_{n}\right\|+\left\|z_{n}-t_{n}\right\|
$$

and hence $\left\|S_{n} t_{n}-t_{n}\right\| \rightarrow 0$. At the same time, observe that for all $i \in\{1,2, \ldots\}$,

$$
\begin{aligned}
\left\|S_{i} t_{n}-t_{n}\right\| \leq & \left\|S_{i} t_{n}-S_{i}\left(S_{n} t_{n}\right)\right\|+\left\|S_{i}\left(S_{n} t_{n}\right)-S_{n} t_{n}\right\|\|+\| S_{n} t_{n}-t_{n} \| . \\
& \leq 2\left\|S_{n} t_{n}-t_{n}\right\|+\sup _{x \in K}\left\|S_{i}\left(S_{n} x\right)-S_{n} x\right\| .
\end{aligned}
$$

It follows from (3.8) and the condition $\left(^{*}\right)$ that for all $i \in\{1,2, \ldots\}$,

$$
\lim _{n \rightarrow \infty}\left\|S_{i} t_{n}-t_{n}\right\|=0 .
$$

As $\left\{x_{n}\right\}$ is bounded, there exists a subsequence $\left\{x_{n_{i}}\right\}$ of $\left\{x_{n}\right\}$ such that $x_{n i} \rightarrow w$. From \| $x_{n}-u_{n} \| \rightarrow 0$, we obtain that $u_{n i} \rightarrow w$. From $\left\|u_{n}-t_{n}\right\| \rightarrow 0$, we also obtain that $t_{n i} \rightarrow$ $w$. Since $\left\{u_{n i}\right\} \subset C$ and $C$ is closed and convex, we obtain $w \in C$.

First, we show $w \in \operatorname{GEP}(F, B)$. By $u_{n}=T_{r_{n}}\left(x_{n}-r_{n} B x_{n}\right) \in C$, we know that

$$
F\left(u_{n}, y\right)+\left\langle B x_{n}, y-u_{n}\right\rangle+\frac{1}{r^{n}}\left\langle y-u_{n}, u_{n}-x_{n}\right\rangle \geq 0, \forall y \in C .
$$

It follows from (A2) that

$$
\left\langle B x_{n}, y-u_{n}\right\rangle+\frac{1}{r_{n}}\left\langle y-u_{n}, u_{n}-x_{n}\right\rangle \geq F\left(y, u_{n}\right), \forall y \in C .
$$

Hence,

$$
\left\langle B x_{n_{i}}, y-u_{n_{i}}\right\rangle+\left\langle y-u_{n_{i}}, \frac{u_{n_{i}}-x_{n_{i}}}{r_{n_{i}}}\right\rangle \geq F\left(y, u_{n_{i}}\right), \forall y \in C .
$$

For $t$ with $0<t \leq 1$ and $y \in C$, let $y_{t}=t_{y}+(1-t) w$. Since $y \in C$ and $w \in C$, we obtain $y_{t} \in C$. So, from (3.10) we have

$$
\begin{aligned}
\left\langle y_{t}-u_{n_{i}}, B y_{t}\right\rangle \geq\left\langle y_{t}-u_{n_{i}}, B y_{t}\right\rangle-\left\langle y_{t}-u_{n_{i}}, B x_{n_{i}}\right\rangle & \\
- & \left\langle y_{t}-u_{n_{i}}, \frac{u_{n_{i}}-x_{n_{i}}}{r_{n_{i}}}\right\rangle+F\left(y_{t}, u_{n_{i}}\right) \\
=\left\langle y_{t}\right. & \left.-u_{n_{i}}, B y_{t}-B u_{n_{i}}\right\rangle+\left\langle y_{t}-u_{n_{i}}, B u_{n_{i}}-B x_{n_{i}}\right\rangle \\
& -\left\langle y_{t}-u_{n_{i}}, \frac{u_{n_{i}}-x_{n_{i}}}{r_{n_{i}}}\right\rangle+F\left(y_{t}, u_{n_{i}}\right) .
\end{aligned}
$$

Since $\left\|u_{n_{i}}-x_{n_{i}}\right\| \rightarrow 0$, we have $\left\|B u_{n_{i}}-B x_{n_{i}}\right\| \rightarrow 0$. Further, from the inverse-strongly monotonicity of $B$, we have $\left\langle y_{t}-u_{n_{i}}, B y_{t}-B u_{n_{i}}\right\rangle \geq 0$. Hence, from (A4), $\frac{u_{n_{i}}-x_{n_{i}}}{r_{n_{i}}} \rightarrow 0$ and $u_{n_{i}} \rightarrow w$, we have

$$
\left\langle y_{t}-w, B y_{t}\right\rangle \geq F\left(y_{t}, w\right)
$$

as $i \rightarrow \infty$. From (A1), (A4) and (3.11), we also have

$$
\begin{aligned}
0= & F\left(y_{t}, y_{t}\right) \leq t F\left(y_{t}, y\right)+(1-t) F\left(y_{t}, w\right) \\
& \leq t F\left(y_{t}, y\right)+(1-t)\left\langle y_{t}-w, B y_{t}\right\rangle \\
& =t F\left(y_{t}, y\right)+(1-t) t\left\langle y-w, B y_{t}\right\rangle .
\end{aligned}
$$


and hence

$$
0 \leq F\left(y_{t}, \gamma\right)+(1-t)\left\langle y-w, B y_{t}\right\rangle .
$$

Letting $t \rightarrow 0$, we have, for each $y \in C$,

$$
F(w, y)+\langle y-w, B w\rangle \geq 0 .
$$

This implies that $w \in G E P(F, B)$.

We next show that $w \in \cap_{i=1}^{\infty} \operatorname{Fix}\left(S_{i}\right)$. Assume $w \notin \cap_{i=1}^{\infty} \operatorname{Fix}\left(S_{i}\right)$. Since $t_{n_{i}} \rightarrow w$ and $w \neq S_{i_{0}} w$ for some $i_{0} \in\{1,2, \ldots\}$ from the Opial condition, we have

$$
\begin{aligned}
\liminf _{i \rightarrow \infty}\left\|t_{n_{i}}-w\right\| & <\liminf _{i \rightarrow \infty}\left\|t_{n_{i}}-S_{i_{0}} w\right\| \\
& \leq \liminf _{i \rightarrow \infty}\left\{\left\|t_{n_{i}}-S_{i_{0}} t_{n_{i}}\right\|+\left\|S_{i_{0}} t_{n_{i}}-S_{i_{0}} w\right\|\right\} \\
& \leq \liminf _{i \rightarrow \infty}\left\|t_{n_{i}}-w\right\| .
\end{aligned}
$$

This is a contradiction. Hence, we get $w \in \cap_{i=1}^{\infty} \operatorname{Fix}\left(S_{i}\right)$.

Finally we show $w \in V I(C, A)$. Let

$$
T v= \begin{cases}A v+N_{C} v & \text { if } v \in C, \\ \varnothing & \text { if } v \in C .\end{cases}
$$

where $N_{C} v$ is the normal cone to $C$ at $v \in C$. We have already mentioned that in this case the mapping $T$ is maximal monotone, and $0 \in T v$ if and only if $v \in V I(C, A)$. Let $(v, g) \in G(T)$. Then $T v=A v+N_{C} v$ and hence $g-A v \in N_{C} v$.

Hence, we have $\langle v-t, g-A v\rangle \geq 0$ for all $t \in C$. On the other hand, from $t_{n}=P_{C}\left(u_{n}-\right.$ $\left.\lambda_{n} A y_{n}\right)$ and $v \in C$, we have

$$
\left\langle u_{n}-\lambda_{n} A y_{n}-t_{n}, t_{n}-v\right\rangle \geq 0
$$

and hence

$$
\left\langle v-t_{n}, \frac{t_{n}-u_{n}}{\lambda_{n}}+A y_{n}\right\rangle \geq 0 .
$$

Therefore, we have

$$
\begin{aligned}
\left\langle v-t_{n_{i}}, g\right\rangle & \geq\left\langle v-t_{n_{i}}, A v\right\rangle \\
& \geq\left\langle v-t_{n^{\prime}}, A v\right\rangle-\left\langle v-t_{n_{i}}, \frac{t_{n_{i}}-u_{n_{i}}}{\lambda_{n_{i}}}+A y_{n_{i}}\right\rangle \\
& =\left\langle v-t_{n_{i}}, A v-A y_{n_{i}}-\frac{t_{n_{i}}-u_{n_{i}}}{\lambda_{n_{i}}}\right\rangle \\
& =\left\langle v-t_{n_{i}}, A v-A t_{n_{i}}+A t_{n_{i}}-A y_{n_{i}}-\frac{t_{n_{i}}-u_{n_{i}}}{\lambda_{n_{i}}}\right\rangle \\
& =\left\langle v-t_{n_{i^{\prime}}}, A v-A t_{n_{i}}\right\rangle+\left\langle v-t_{n_{i}}, A t_{n_{i}}-A y_{n_{i}}\right\rangle-\left\langle v-t_{n_{i^{\prime}}} \frac{t_{n_{i}}-u_{n_{i}}}{\lambda_{n_{i}}}\right\rangle \\
& \geq\left\langle v-t_{n_{i}}, A t_{n_{i}}-A y_{n_{i}}\right\rangle-\left\langle v-t_{n_{i}}, \frac{t_{n_{i}}-u_{n_{i}}}{\lambda_{n_{i}}}\right\rangle
\end{aligned}
$$

Hence, we obtain $\langle v-w, g\rangle \geq 0$ as $i \rightarrow \infty$. Since $T$ is maximal monotone, we have $w$ $\in T^{-1} 0$ and hence $w \in V I(C, A)$. This implies that $w \in \Omega$. 
From $l_{0}=P_{\Omega} x, w \in \Omega$ and (3.6), we have

$$
\left\|l_{0}-x\right\| \leq\|w-x\| \leq \liminf _{i \rightarrow \infty}\left\|x_{n_{i}}-x\right\| \leq \limsup _{i \rightarrow \infty}\left\|x_{n_{i}}-x\right\| \leq\left\|l_{0}-x\right\| .
$$

Hence, we obtain

$$
\lim _{i \rightarrow \infty}\left\|x_{n_{i}}-x\right\|=\|w-x\|
$$

From $x_{n_{i}}-x \rightarrow w-x$, we have $x_{n_{i}}-x \rightarrow w-x$, and hence $x_{n_{i}} \rightarrow w$. Since $x_{n}=P_{Q_{n}} x$ and $l_{0} \in \Omega \subset C_{n} \cap Q_{n} \subset Q_{n}$, we have

$$
-\left\|l_{0}-x_{n_{i}}\right\|^{2} \leq\left\langle l_{0}-x_{n_{i}}, x_{n_{i}}-x\right\rangle+\left\langle l_{0}-x_{n_{i}}, x-l_{0}\right\rangle \geq\left\langle l_{0}-x_{n_{i}}, x-l_{0}\right\rangle .
$$

As $i \rightarrow \infty$, we obtain - $\left\|l_{0}-w\right\|^{2} \geq\left\langle l_{0}-w, x-l_{0}\right\rangle \geq 0$ by $l_{0}=P_{\Omega} x$ and $w \in \Omega$. Hence, we have $w=l_{0}$. This implies that $x_{n} \rightarrow l_{0}$. It is easy to see $u_{n} \rightarrow l_{0}, y_{n} \rightarrow l_{0}$ and $z_{n} \rightarrow$ $l_{0}$. The proof is now complete.

By combining the arguments in the proof of Theorem 3.1 and those in the proof of Theorem 3.1 in [3], we can easily obtain the following weak convergence theorem for an iterative algorithm based on the extragradient method which solves the problem of finding a common element of the set of solutions of a generalized equilibrium problem, the set of fixed points of a family of infinitely nonexpansive mappings and the set of solutions of the variational inequality for a monotone, Lipschitz-continuous mapping in a Hilbert space.

Theorem 3.2. Let $C$ be a nonempty closed convex subset of a real Hilbert space $H$. Let $F$ be a bifunction from $C \times C$ to $R$ satisfying (A1)-(A4). Let $A$ be a monotone, and $k$-Lipschitz-continuous mapping of $C$ into $H$ and $B$ be an $\alpha$-inverse-strongly monotone mapping of $C$ into $H$. Let $S_{1}, S_{2}, \ldots$ be a family of infinitely nonexpansive mappings of $C$ into itself such that $\Omega=\cap_{i=1}^{\infty} \operatorname{Fix}\left(S_{i}\right) \cap \operatorname{VI}(C, A) \cap \operatorname{GEP}(F, B) \neq \emptyset$. Assume that for all $i \in$ $\{1,2, \ldots\}$ and for any bounded subset $K$ of $C$, thenthere holds

$$
\lim _{n \rightarrow \infty} \sup _{x \in K}\left\|S_{n} x-S_{i}\left(S_{n} x\right)\right\|=0 .
$$

Let $\left\{x_{n}\right\},\left\{u_{n}\right\}$ and $\left\{y_{n}\right\}$ be the sequences generated by

$$
\left\{\begin{array}{l}
x_{1}=x \in C, \\
F\left(u_{n}, y\right)+\left\langle B x_{n}, y-u_{n}\right\rangle+\frac{1}{r_{n}}\left\langle y-u_{n}, u_{n}-x_{n}\right\rangle \geq 0, \quad \forall y \in C, \\
y_{n}=P_{C}\left(u_{n}-\lambda_{n} A u_{n}\right), \\
x_{n+1}=\beta_{n} x_{n}+\left(1-\beta_{n}\right) S_{n} P_{C}\left(u_{n}-\lambda_{n} A y_{n}\right)
\end{array}\right.
$$

for every $n=1,2, \ldots$ If $\left\{\lambda_{n}\right\} \subset[a, b]$ for some $a, b \in\left(0, \frac{1}{k}\right),\left\{\beta_{n}\right\} \subset[\delta, \varepsilon]$ for some $\delta, \varepsilon$ $\in(0,1)$ and $\left\{r_{n}\right\} \subset[d, e]$ for some $d, e \in(0,2 \alpha)$. Then, $\left\{x_{n}\right\},\left\{u_{n}\right\}$ and $\left\{y_{n}\right\}$ converge weakly to $w \in \Omega$, where $w=\lim _{n \rightarrow \infty} P_{\Omega} x_{n}$.

\section{Applications}

By Theorems 3.1 and 3.2, we can obtain many new and interesting convergence theorems in a real Hilbert space. We give some examples as follows:

Let $A=0$, by Theorems 3.1 and 3.2, respectively, we obtain the following results.

Theorem 4.1. Let $C$ be a nonempty closed convex subset of a real Hilbert space $H$. Let $F$ be a bifunction from $C \times C$ to $R$ satisfying (A1)-(A4). Let $B$ be an $\alpha$-inversestrongly monotone mapping of $C$ into $H$. Let $S_{1}, S_{2}, \ldots$ be a family of infinitely 
nonexpansive mappings of $C$ into itself such that $\sum=\cap_{i=1}^{\infty} \operatorname{Fix}\left(S_{i}\right) \cap \operatorname{GEP}(F, B) \neq \emptyset$. Assume that for all $i \in\{1,2, \ldots\}$ and for any bounded subset $K$ of $C$, thenthere holds

$$
\lim _{n \rightarrow \infty} \sup _{x \in K}\left\|S_{n} x-S_{i}\left(S_{n} x\right)\right\|=0 .
$$

Let $\left\{x_{n}\right\},\left\{u_{n}\right\}\left\{y_{n}\right\}$, and $\left\{z_{n}\right\}$ be the sequences generated by

$$
\left\{\begin{array}{l}
x_{1}=x \in C, \\
F\left(u_{n}, y\right)+\left\langle B x_{n}, y-u_{n}\right\rangle+\frac{1}{r_{n}}\left\langle y-u_{n}, u_{n}-x_{n}\right\rangle \geq 0, \quad \forall y \in C, \\
z_{n}=\left(1-\alpha_{n}-\beta_{n}\right) x_{n}+\alpha_{n} u_{n}+\beta_{n} S_{n} u_{n} \\
C_{n}=\left\{z \in C:\left\|z_{n}-z\right\|^{2} \leq\left\|x_{n}-z\right\|^{2}\right\} \\
Q_{n}=\left\{z \in C:\left\langle x_{n}-z, x-x_{n}\right\rangle \geq 0\right\} \\
x_{n+1}=P_{C_{n} \cap Q_{n}} x
\end{array}\right.
$$

for every $n=1,2, \ldots$ where $\left\{r_{n}\right\} \subset[d, e]$ for some $d, e \in(0,2 \alpha)$, and $\left\{\alpha_{n}\right\},\left\{\beta_{n}\right\}$ are sequences in $[0,1]$ satisfying the conditions:

(i) $\alpha_{n}+\beta_{n} \leq 1$ for all $n \in N$;

(ii) $\lim _{n \rightarrow \infty} \alpha_{n}=0$;

(iii) $\liminf _{n \rightarrow \infty} \beta_{n}>0$ for all $n \in N$;

Then, $\left\{x_{n}\right\}$, $\left\{u_{n}\right\}$, and $\left\{z_{n}\right\}$ converge strongly to $w=P_{\Sigma}(x)$.

Theorem 4.2. Let $C$ be a nonempty closed convex subset of a real Hilbert space $H$. Let $F$ be a bifunction from $C \times C$ to $R$ satisfying (A1)-(A4). Let $B$ be an $\alpha$-inversestrongly monotone mapping of $C$ into $H$. Let $S_{1}, S_{2}, \ldots$ be a family of infinitely nonexpansive mappings of $C$ into itself such that $\sum=\cap_{i=1}^{\infty} \operatorname{Fix}\left(S_{i}\right) \cap \operatorname{GEP}(F, B) \neq \emptyset$. Assume that for all $i \in\{1,2, \ldots\}$ and for any bounded subset $K$ of $C$, thenthere holds

$$
\lim _{n \rightarrow \infty} \sup _{x \in K}\left\|S_{n} x-S_{i}\left(S_{n} x\right)\right\|=0 .
$$

Let $\left\{x_{n}\right\}$ and $\left\{u_{n}\right\}$ be sequences generated by

$$
\left\{\begin{array}{l}
x_{1}=x \in C, \\
F\left(u_{n}, y\right)+\left\langle B x_{n}, y-u_{n}\right\rangle+\frac{1}{r_{n}}\left\langle y-u_{n}, u_{n}-x_{n}\right\rangle \geq 0, \quad \forall y \in C, \\
x_{n+1}=\beta_{n} x_{n}+\left(1-\beta_{n}\right) S_{n} u_{n}
\end{array}\right.
$$

for every $n=1,2, \ldots$ If $\left\{\beta_{n}\right\} \subset[\delta, \varepsilon]$ for some $\delta, \varepsilon \in(0,1)$ and $\left\{r_{n}\right\} \subset[d, e]$ for some $d, e \in(0,2 \alpha)$. Then, $\left\{x_{n}\right\}$ and $\left\{u_{n}\right\}$ converge weakly to $w \in \Sigma$, where $w=\lim _{n \rightarrow \infty} P_{\Sigma} x_{n}$.

Theorem 4.3. Let $C$ be a nonempty closed convex subset of a real Hilbert space $H$. Let $F$ be a bifunction from $C \times C$ to $R$ satisfying (A1)-(A4). Let $A$ be a monotone and $k$ Lipschitz-continuous mapping of $C$ into $H$ and $B$ be an $\alpha$-inverse-strongly monotone mapping of $C$ into $H$. Let $S_{1}, S_{2}, \ldots$ be a family of infinitely nonexpansive mappings of $C$ into itself such that $\Omega=\cap_{i=1}^{\infty} \operatorname{Fix}\left(S_{i}\right) \cap \operatorname{VI}(C, A) \cap \operatorname{GEP}(F, B) \neq \emptyset$. Assume that for all $i \in$ $\{1,2, \ldots\}$ and for any bounded subset $K$ of $C$, thenthere holds

$$
\lim _{n \rightarrow \infty} \sup _{x \in K}\left\|S_{n} x-S_{i}\left(S_{n} x\right)\right\|=0 .
$$


Let $\left\{x_{n}\right\},\left\{u_{n}\right\},\left\{y_{n}\right\}$, and $\left\{z_{n}\right\}$ be sequences generated by

$$
\left\{\begin{array}{l}
x_{1}=x \in C, \\
F\left(u_{n}, y\right)+\left\langle B x_{n}, y-u_{n}\right\rangle+\frac{1}{r_{n}}\left\langle y-u_{n}, u_{n}-x_{n}\right\rangle \geq 0, \quad \forall y \in C, \\
y_{n}=P_{C}\left(u_{n}-\lambda_{n} A u_{n}\right), \\
z_{n}=\left(1-\beta_{n}\right) x_{n}+\beta_{n} S_{n} P_{C}\left(u_{n}-\lambda_{n} A y_{n}\right), \\
C_{n}=\left\{z \in C:\left\|z_{n}-z\right\|^{2} \leq\left\|x_{n}-z\right\|^{2},\right. \\
Q_{n}=\left\{z \in C:\left\langle x_{n}-z, x-x_{n}\right\rangle \geq 0\right\}, \\
x_{n+1}=P_{C_{n} \cap Q_{n}} x
\end{array}\right.
$$

for every $n=1,2, \ldots$ where $\left\{\lambda_{n}\right\} \subset[a, b]$ for some $a, b \in\left(0, \frac{1}{4 k}\right),\left\{r_{n}\right\} \subset[d, e]$ for some $d, e \in(0,2 \alpha)$, and $\left\{\beta_{n}\right\}$ is a sequence in $[0,1]$ satisfying $\liminf _{n \rightarrow \infty} \beta_{n}>0$. Then, $\left\{x_{n}\right\}$, $\left\{u_{n}\right\},\left\{y_{n}\right\}$, and $\left\{z_{n}\right\}$ converge strongly to $w=P_{\Omega}(x)$.

Proof. Putting $\gamma_{n}=1$ and $\alpha_{n}=0$, by Theorem 3.1, we obtain the desired result.

Let $B=0$, by Theorems 3.1, 3.2, and 4.3, we obtain the following results.

Theorem 4.4. Let $C$ be a nonempty closed convex subset of a real Hilbert space $H$. Let $F$ be a bifunction from $C \times C$ to $R$ satisfying (A1)-(A4). Let $A$ be a monotone and $k$ Lipschitz-continuous mapping of $C$ into $H$. Let $S_{1}, S_{2}, \ldots$ be a family of infinitely nonexpansive mappings of $C$ into itself such that $\Lambda=\cap_{i=1}^{\infty} \operatorname{Fix}\left(S_{i}\right) \cap \operatorname{VI}(C, A) \cap E P(F) \neq \emptyset$. Assume that for all $i \in\{1,2, \ldots\}$, and for any bounded subset $K$ of $C$, there holds

$$
\lim _{n \rightarrow \infty} \sup _{x \in K}\left\|S_{n} x-S_{i}\left(S_{n} x\right)\right\|=0 .
$$

Let $\left\{x_{n}\right\},\left\{u_{n}\right\},\left\{y_{n}\right\}$, and $\left\{z_{n}\right\}$ be the sequences generated by

$$
\left\{\begin{array}{l}
x_{1}=x \in C, \\
F\left(u_{n}, y\right)+\frac{1}{r_{n}}\left\langle y-u_{n}, u_{n}-x_{n}\right\rangle \geq 0, \quad \forall y \in C, \\
y_{n}=\left(1-\gamma_{n}\right) u_{n}+\gamma_{n} P_{C}\left(u_{n}-\lambda_{n} A u_{n}\right) \\
z_{n}=\left(1-\alpha_{n}-\beta_{n}\right) x_{n}+\alpha_{n} y_{n}+\beta_{n} S_{n} P_{C}\left(u_{n}-\lambda_{n} A y_{n}\right) \\
C_{n}=\left\{z \in C:\left\|z_{n}-z\right\|^{2} \leq\left\|x_{n}-z\right\|^{2}+\left(3-3 \gamma_{n}+\alpha_{n}\right) b^{2}\left\|A u_{n}\right\|^{2}\right\} \\
Q_{n}=\left\{z \in C:\left\langle x_{n}-z, x-x_{n}\right\rangle \geq 0\right\} \\
x_{n+1}=P_{C_{n} \cap Q_{n}} x
\end{array}\right.
$$

for every $n=1,2, \ldots$ where $\left\{\lambda_{n}\right\} \subset[a, b]$ for some $a, b \in\left(0, \frac{1}{4 k}\right),\left\{r_{n}\right\} \subset[d,+\infty)$ for some $d>0$, and $\left\{\alpha_{n}\right\},\left\{\beta_{n}\right\},\left\{\gamma_{n}\right\}$ are three sequences in $[0,1]$ satisfying the following conditions:

(i) $\alpha_{n}+\beta_{n} \leq 1$ for all $n \in N$;

(ii) $\lim _{n \rightarrow \infty} \alpha_{n}=0$;

(iii) $\liminf _{n \rightarrow \infty} \beta_{n}>0$;

(iv) $\lim _{n \rightarrow \infty} \gamma_{n}=1$ and $\gamma_{n}>\frac{3}{4}$ for all $n \in N$;

Then, $\left\{x_{n}\right\},\left\{u_{n}\right\},\left\{y_{n}\right\}$ and $\left\{z_{n}\right\}$ converge strongly to $w=P_{\Lambda}(x)$.

Theorem 4.5. Let $C$ be a nonempty closed convex subset of a real Hilbert space $H$. Let $F$ be a bifunction from $C \times C$ to $R$ satisfying (A1)-(A4). Let $A$ be a monotone and $k$ Lipschitz-continuous mapping of $C$ into $H$. Let $S_{1}, S_{2}, \ldots$ be a family of infinitely nonexpansive mappings of $C$ into itself such that $\Lambda=\cap_{i=1}^{\infty} \operatorname{Fix}\left(S_{i}\right) \cap V I(C, A) \cap E P(F) \neq \emptyset$. Assume that for all $i \in\{1,2, \ldots\}$ and for any bounded subset $K$ of $C$, thenthere holds 


$$
\lim _{n \rightarrow \infty} \sup _{x \in K}\left\|S_{n} x-S_{i}\left(S_{n} x\right)\right\|=0 .
$$

Let $\left\{x_{n}\right\},\left\{u_{n}\right\}$, and $\left\{y_{n}\right\}$ be the sequences generated by

$$
\left\{\begin{array}{l}
x_{1}=x \in C, \\
F\left(u_{n}, y\right)+\frac{1}{r_{n}}\left\langle y-u_{n}, u_{n}-x_{n}\right\rangle \geq 0, \quad \forall y \in C, \\
y_{n}=P_{C}\left(u_{n}-\lambda_{n} A u_{n}\right), \\
x_{n+1}=\beta_{n} x_{n}+\left(1-\beta_{n}\right) S_{n} P_{C}\left(u_{n}-\lambda_{n} A y_{n}\right)
\end{array}\right.
$$

for every $n=1,2, \ldots$. If $\left\{\lambda_{n}\right\} \subset[a, b]$ for some $a, b \in\left(0, \frac{1}{k}\right),\left\{\beta_{n}\right\} \subset[\delta, \varepsilon]$, for some $\delta, \varepsilon$ $\in(0,1)$ and $\left\{r_{n}\right\} \subset[d,+\infty]$ for some $d>0$, then $\left\{x_{n}\right\},\left\{u_{n}\right\}$ and $\left\{y_{n}\right\}$ converge weakly to $w \in \Lambda$, where $w=\lim _{n \rightarrow \infty} P_{\Lambda} x_{n}$.

Theorem 4.6. Let $C$ be a nonempty closed convex subset of a real Hilbert space $H$. Let $F$ be a bifunction from $C \times C$ to $R$ satisfying (A1)-(A4). Let $A$ be a monotone and $k$-Lipschitz-continuous mapping of $C$ into $H$. Let $S_{1}, S_{2}, \ldots$ be a family of infinitely nonexpansive mappings of $C$ into itself such that $\Lambda=\cap_{i=1}^{\infty} \operatorname{Fix}\left(S_{i}\right) \cap \operatorname{VI}(C, A) \cap E P(F) \neq \emptyset$. Assume that for all $i \in\{1,2, \ldots\}$ and for any bounded subset $K$ of $C$, thenthere holds

$$
\lim _{n \rightarrow \infty} \sup _{x \in K}\left\|S_{n} x-S_{i}\left(S_{n} x\right)\right\|=0 .
$$

Let $\left\{x_{n}\right\},\left\{u_{n}\right\}\left\{y_{n}\right\}$, and $\left\{z_{n}\right\}$ be the sequences generated by

$$
\left\{\begin{array}{l}
x_{1}=x \in C, \\
F\left(u_{n}, y\right)+\frac{1}{r_{n}}\left\langle y-u_{n}, u_{n}-x_{n}\right\rangle \geq 0, \quad \forall y \in C, \\
y_{n}=P_{C}\left(u_{n}-\lambda \lambda_{n} A u_{n}\right), \\
z_{n}=\left(1-\beta_{n}\right) x_{n}+\beta_{n} S_{n} P_{C}\left(u_{n}-\lambda_{n} A y_{n}\right), \\
C_{n}=\left\{z \in C:\left\|z_{n}-z\right\|^{2} \leq\left\|x_{n}-z\right\|^{2},\right. \\
Q_{n}=\left\{z \in C:\left\langle x_{n}-z_{1} x-x_{n}\right\rangle \geq 0\right\} \\
x_{n+1}=P_{C_{n} \cap Q_{n}} x
\end{array}\right.
$$

for every $n=1,2, \ldots$. where $\left\{\lambda_{n}\right\} \subset[a, b]$ for some $a, b \in\left(0, \frac{1}{4 k}\right),\left\{r_{n}\right\} \subset[d,+\infty)$ and for some $d>0$, and $\left\{\beta_{n}\right\}$ is a sequence in $[0,1]$ satisfying $\liminf _{n \rightarrow \infty} \beta_{n}>0$. Then, $\left\{x_{n}\right\}$, $\left\{u_{n}\right\},\left\{y_{n}\right\}$, and $\left\{z_{n}\right\}$ converge strongly to $w=P_{\Lambda}(x)$.

Let $B=0$ and $F(x, y)=0$ for $x, y \in C$, by Theorems 3.1 and 4.3, we obtain the following results.

Theorem 4.7. Let $C$ be a nonempty closed convex subset of a real Hilbert space $H$. Let $A$ be a monotone and $k$-Lipschitz-continuous mapping of $C$ into $H$. Let $S_{1}, S_{2}, \ldots$ be a family of infinitely nonexpansive mappings of $C$ into itself such that $\Gamma=\cap_{i=1}^{\infty} \operatorname{Fix}\left(S_{i}\right) \cap \operatorname{VI}(C, A) \neq \emptyset$. Assume that for all $i \in\{1,2, \ldots\}$ and for any bounded subset $K$ of $C$, thenthere holds

$$
\lim _{n \rightarrow \infty} \sup _{x \in K}\left\|S_{n} x-S_{i}\left(S_{n} x\right)\right\|=0 .
$$

Let $\left\{x_{n}\right\},\left\{y_{n}\right\}$, and $\left\{z_{n}\right\}$ be the sequences generated by

$$
\left\{\begin{array}{l}
x_{1}=x \in C \\
y_{n}=\left(1-\gamma_{n}\right) x_{n}+\gamma_{n} P_{C}\left(x_{n}-\lambda_{n} A x_{n}\right) \\
z_{n}=\left(1-\alpha_{n}-\beta_{n}\right) x_{n}+\alpha_{n} y_{n}+\beta_{n} S_{n} P_{C}\left(x_{n}-\lambda_{n} A y_{n}\right) \\
C_{n}=\left\{z \in C:\left\|z_{n}-z\right\|^{2} \leq\left\|x_{n}-z\right\|^{2}+\left(3-3 \gamma_{n}+\alpha_{n}\right) b^{2}\left\|A x_{n}\right\|^{2}\right\} \\
Q_{n}=\left\{z \in C:\left\langle x_{n}-z, x-x_{n}\right\rangle \geq 0\right\} \\
x_{n+1}=P_{C_{n} \cap Q_{n}} x
\end{array}\right.
$$


for every $n=1,2, \ldots$ where $\left\{\lambda_{n}\right\} \subset[a, b]$ for some $a, b \in\left(0, \frac{1}{4 k}\right)$, and $\left\{\alpha_{n}\right\},\left\{\beta_{n}\right\},\left\{\gamma_{n}\right\}$ are three sequences in $[0,1]$ satisfying the following conditions:

(i) $\alpha_{n}+\beta_{n} \leq 1$ for all $n \in N$;

(ii) $\lim _{n \rightarrow \infty} \alpha_{n}=0$;

(iii) $\liminf _{n \rightarrow \infty} \beta_{n}>0$;

(iv) $\lim _{n \rightarrow \infty} \gamma_{n}=1$ and $\gamma_{n}>\frac{3}{4}$ for all $n \in N$;

Then, $\left\{x_{n}\right\},\left\{y_{n}\right\}$, and $\left\{z_{n}\right\}$ converge strongly to $w=P_{\Gamma}(x)$.

Theorem 4.8. Let $C$ be a nonempty closed convex subset of a real Hilbert space $H$. Let $A$ be a monotone and $k$-Lipschitz-continuous mapping of $C$ into $H$. Let $S_{1}, S_{2}, \ldots$ be a family of infinitely nonexpansive mappings of $C$ into itself such that $\Gamma=\cap_{i=1}^{\infty} \operatorname{Fix}\left(S_{i}\right) \cap \operatorname{VI}(C, A) \neq \emptyset$. Assume that for all $i \in\{1,2, \ldots\}$ and for any bounded subset $K$ of $C$, thenthere holds

$$
\lim _{n \rightarrow \infty} \sup _{x \in K}\left\|S_{n} x-S_{i}\left(S_{n} x\right)\right\|=0 .
$$

Let $\left\{x_{n}\right\},\left\{y_{n}\right\}$, and $\left\{z_{n}\right\}$ be the sequences generated by

$$
\left\{\begin{array}{l}
x_{1}=x \in C, \\
y_{n}=P_{C}\left(x_{n}-\lambda_{n} A x_{n}\right), \\
z_{n}=\left(1-\beta_{n}\right) x_{n}+\beta_{n} S_{n} P_{C}\left(x_{n}-\lambda_{n} A y_{n}\right), \\
C_{n}=\left\{z \in C:\left\|z_{n}-z\right\|^{2} \leq\left\|x_{n}-z\right\|^{2},\right. \\
Q_{n}=\left\{z \in C:\left\langle x_{n}-z, x-x_{n}\right\rangle \geq 0\right\}, \\
x_{n+1}=P_{C_{n} \cap Q_{n}} x
\end{array}\right.
$$

for every $n=1,2, \ldots$. where $\left\{\lambda_{n}\right\} \subset[a, b]$ for some $a, b \in\left(0, \frac{1}{4 k}\right)$, and $\left\{\beta_{n}\right\}$ is a sequence in $[0,1]$ satisfying $\liminf _{n \rightarrow \infty} \beta_{n}>0$. Then, $\left\{x_{n}\right\},\left\{y_{n}\right\}$, and $\left\{z_{n}\right\}$ converge strongly to $w=P_{\Gamma}(x)$.

Let $F(x, y)=0$ for $x, y \in C$, then by Theorem 3.2 and the proof of Theorem 4.7 in [3], we obtain the following result.

Theorem 4.9. Let $C$ be a nonempty closed convex subset of a real Hilbert space $H$. Let $A$ be a monotone and $k$-Lipschitz-continuous mapping of $C$ into $H$ and $B$ be an $\alpha$ inverse-strongly monotone mapping of $C$ into $H$. Let $S_{1}, S_{2}, \ldots$ be a family of infinitely nonexpansive mappings of $C$ into itself such that $\Xi=\cap_{i=1}^{\infty} \operatorname{Fix}\left(S_{i}\right) \cap \operatorname{VI}(C, A) \cap \operatorname{VI}(C, B) \neq \emptyset$. Assume that for all $i \in\{1,2, \ldots\}$ and for any bounded subset $K$ of $C$, thenthere holds

$$
\lim _{n \rightarrow \infty} \sup _{x \in K}\left\|S_{n} x-S_{i}\left(S_{n} x\right)\right\|=0 .
$$

Let $\left\{x_{n}\right\},\left\{u_{n}\right\}$, and $\left\{y_{n}\right\}$ be the sequences generated by

$$
\left\{\begin{array}{l}
x_{1}=x \in C \\
u_{n}=P_{C}\left(x_{n}-r_{n} B x_{n}\right), \\
y_{n}=P_{C}\left(u_{n}-\lambda_{n} A u_{n}\right), \\
x_{n+1}=\alpha_{n} x_{n}+\left(1-\alpha_{n}\right) S_{n} P_{C}\left(u_{n}-\lambda_{n} A y_{n}\right)
\end{array}\right.
$$


for every $n=1,2, \ldots$ if $\left\{\lambda_{n}\right\} \subset[a, b]$ for some $a, b \in\left(0, \frac{1}{k}\right),\left\{\beta_{n}\right\} \subset[\delta, \varepsilon]$ for some $\delta, \varepsilon$ $\in(0,1)$ and $\left\{r_{n}\right\} \subset[d, e]$ for some $d, e \in(0,2 \alpha)$. Then, $\left\{x_{n}\right\}$ and $\left\{u_{n}\right\}$ converge weakly to $w \in \Xi$, where $w=\lim _{n \rightarrow \infty} P_{\Xi} x_{n}$.

\section{Remark 4.1.}

(i) For all $n \geq 1$, let $S_{n}=S$ be a nonexpansive mapping, by Theorems 3.2, 4.2, 4.7, 4.8, and 4.9 we recover Theorem 3.1 in [5], Theorem 3.1 in [1], Theorem 5 in [26], Theorem 3.1 in [23], and Theorem 4.7 in [3]. In addition, let $A=0$, by Theorems 4.6 and 4.5, respectively, we recover Theorems 3.1 and 4.1 in [11].

(ii) For all $n \geq 1$, let $S_{n}=S$ be a nonexpansive mapping, by Theorems 3.1, 4.3, and 4.4, respectively, we recover Theorems 4.3, 4.4, and 4.7 in [4] with some modified conditions on $F$.

(iii) Theorems 3.1, 3.2, 4.3-4.7 also improve the main results in $[10,12,13]$ because the inverse strongly monotonicity of $A$ has been replaced by the monotonicity and Lipschitz continuity of $A$.

The following result illustrates that there are the nonexpansive mappings $S_{1}, S_{2}, \ldots$ satisfying the condition (*).

Lemma 4.1. Let $C$ be a nonempty closed convex subset of a real Hilbert space $H$. Let $T$ be a nonexpansive mapping of $C$ into itself such that $\operatorname{Fix}(T) \neq \varnothing$. If we define $S_{n}(x)=\frac{1}{n} \sum_{j=0}^{n-1} T^{j} x$ for $n \in\{1,2, \ldots\}$, and $x \in C$, then the following results hold:

(a) For any bounded subset $K$ of $C$, there holds

$$
\lim _{n \rightarrow \infty} \sup _{x \in K}\left\|S_{n} x-T\left(S_{n} x\right)\right\|=0 .
$$

(b) $\cap_{i=1}^{\infty} \operatorname{Fix}\left(S_{i}\right)=\operatorname{Fix}(T)$.

(c) for all $i \in\{1,2, \ldots\}$ and for any bounded subset $K$ of $C$, there holds

$$
\lim _{n \rightarrow \infty} \sup _{x \in K}\left\|S_{n} x-S_{i}\left(S_{n} x\right)\right\|=0 .
$$

\section{Proof.}

(a) It is due to Bruck [27,28] (please also see Lemma 3.1 in [22]).

(b) It follows from (a) that $\cap_{i=1}^{\infty} \operatorname{Fix}\left(S_{i}\right) \subseteq \operatorname{Fix}(T)$.

Moreover, it is obvious that $\cap_{i=1}^{\infty} \operatorname{Fix}\left(S_{i}\right) \supseteq \operatorname{Fix}(T)$. Hence, $\cap_{i=1}^{\infty} \operatorname{Fix}\left(S_{i}\right)=\operatorname{Fix}(T)$.

(c) It can be proved by mathematical induction. In fact, it is clear that this conclusion holds for $i=1$. Assume that the conclusion holds for $i=m$, that is, for any bounded subset $K$ of $C$, there holds

$$
\lim _{n \rightarrow \infty} \sup _{x \in K}\left\|S_{n} x-S_{m}\left(S_{n} x\right)\right\|=0
$$

We now prove that the conclusion also holds for $i=m+1$. In fact, we observe that

$$
\begin{aligned}
& \lim _{n \rightarrow \infty} \sup _{x \in K}\left\|S_{n} x-S_{m+1}\left(S_{n} x\right)\right\| \leq \lim _{n \rightarrow \infty} \sup _{x \in K}\left\|S_{n} x-S_{m}\left(S_{n} x\right)\right\|+\lim _{n \rightarrow \infty} \sup _{x \in K}\left\|S_{m}\left(S_{n} x\right)-S_{m+1}\left(S_{n} x\right)\right\| \\
& \leq \lim _{n \rightarrow \infty} \sup _{x \in K}\left\|S_{n} x-S_{m}\left(S_{n} x\right)\right\|+\lim _{n \rightarrow \infty} \sup _{x \in K}\left[\frac{1}{m+1}\left\|T^{m}\left(S_{n} x\right)\right\|+\frac{1}{m(m+1)} \sum_{j=0}^{m-1}\left\|T^{j}\left(S_{n} x\right)\right\|\right] .
\end{aligned}
$$


It is easy to verify that $S_{1}, S_{2}, \ldots$ are nonexpansive mappings. It follows from (4.1) and (4.2) that for any bounded subset $K$ of $C$, there holds

$$
\lim _{n \rightarrow \infty} \sup _{x \in K}\left\|S_{n} x-S_{m+1}\left(S_{n} x\right)\right\|=0 .
$$

From Lemma 4.1, we know that by Theorems 3.1 and 3.2, respectively, we can obtain the following results.

Theorem 4.10. Let $C$ be a nonempty closed convex subset of a real Hilbert space $H$. Let $F$ be a bifunction from $C \times C$ to $R$ satisfying (A1)-(A4). Let $A$ be a monotone and $k$-Lipschitz-continuous mapping of $C$ into $H$ and $B$ be an $\alpha$-inverse-strongly monotone mapping of $C$ into $H$. Let $T$ be a nonexpansive mapping of $C$ into itself such that $\Theta=$ $\operatorname{Fix}(T) \cap V I(C, A) \cap G E P(F, B) \neq \varnothing$. Let $\left\{\lambda_{n}\right\} \subset[a, b]$ for some $a, b \in\left(0, \frac{1}{4 k}\right),\left\{r_{n}\right\} \subset[d, e]$ and for some $d, e \in(0,2 \alpha)$, and $\left\{\alpha_{n}\right\},\left\{\beta_{n}\right\}$, and $\left\{\gamma_{n}\right\}$ be three sequences in $[0,1]$ satisfying the following conditions:

(i) $\alpha_{n}+\beta_{n} \leq 1$ for all $n \in N$;

(ii) $\lim _{n \rightarrow \infty} \alpha_{n}=0$;

(iii) $\liminf _{n \rightarrow \infty} \beta_{n}>0$;

(iv) $\lim _{n \rightarrow \infty} \gamma_{n}=1$ and $\gamma_{n}>\frac{3}{4}$ for all $n \in N$; If we define $S_{n}(x)=\frac{1}{n} \sum_{j=0}^{n-1} T^{j} x$ for $n \in$ $\{1,2, \ldots\}$, and $x \in C$, then the sequences $\left\{x_{n}\right\},\left\{u_{n}\right\},\left\{y_{n}\right\}$, and $\left\{z_{n}\right\}$ generated by algorithm (3.1) converge strongly to $w=P_{\Theta}(x)$.

Theorem 4.11. Let $C$ be a nonempty closed convex subset of a real Hilbert space $H$. Let $F$ be a bifunction from $C \times C$ to $R$ satisfying (A1)-(A4). Let $A$ be a monotone and $k$-Lipschitz-continuous mapping of $C$ into $H$ and $B$ be an $\alpha$-inverse-strongly monotone mapping of $C$ into $H$, and $T$ be a nonexpansive mapping of $C$ into itself such that $\Theta=$ $\operatorname{Fix}(T) \cap V I(C, A) \cap G E P(F, B) \neq \varnothing$. Assume that $\left\{\lambda_{n}\right\} \subset[a, b]$ for some $a, b \in\left(0, \frac{1}{k}\right)\left\{\beta_{n}\right\}$ $\subset[\delta, \varepsilon]$ for some $\delta, \varepsilon \in(0,1)$, and $\left\{r_{n}\right\} \subset[d, e]$ some $d, e \in(0,2 \alpha)$. If we define $S_{n}(x)=\frac{1}{n} \sum_{j=0}^{n-1} T^{j} x$ for $n \in\{1,2, \ldots\}$ and $x \in C$, then the sequences $\left\{x_{n}\right\},\left\{u_{n}\right\}$, and $\left\{y_{n}\right\}$ generated by algorithm (3.12) converge weakly to $w \in \Theta$, where $w=\lim _{n \rightarrow \infty} P_{\Theta} x_{n}$.

\section{Competing interests}

The authors declare that they have no competing interests.

\section{Acknowledgements}

This research was supported by the National Natural Science Foundation of China, the Natural Science Foundation of Chongqing (Grant No. CSTC, 2009BB8240), and the Special Fund of Chongqing Key Laboratory (CSTC). The author is grateful to the referees for their detailed comments and helpful suggestions, which have improved the presentation of this article.

Received: 30 November 2010 Accepted: 29 June 2011 Published: 29 June 2011

\section{References}

1. Moudafi, A: Weak convergence theorems for nonexpansive mappings and equilibrium Problems. J Nonlinear Convex Anal. 9, 37-43 (2008)

2. Moudafi, A, Thera, M: Proximal and dynamical approaches to equilibrium problems. Lecture Notes in Econom and Math System, Springer-Verlag, Berlin. 477, 187-201 (1999)

3. Peng, JW, Yao, JC: Weak convergence of an iterative scheme for generalized equilibrium problems. Bull Aust Math Soc. 79, 437-453 (2009). doi:10.1017/S0004972708001378 
4. Peng, JW, Yao, JC: A new hybrid-extragradient method for generalized mixed equilibrium problems and fixed point problems and variational inequality problems. Taiwan J Math. 12(6):1401-1432 (2008)

5. Takahashi, S, Takahashi, W: Strong convergence theorem for a generalized equilibrium problem and a nonexpansive mapping in a Hilbert space. Nonlinear Anal. 69, 1025-1033 (2008). doi:10.1016/j.na.2008.02.042

6. Flam, SD, Antipin, AS: Equilibrium programming using proximal-like algorithms. Math Program. 78, $29-41$ (1997)

7. Blum, E, Oettli, W: From optimization and variational inequalities to equilibrium problems. Math Stud. 63, 123-145 (1994)

8. Combettes, PL, Hirstoaga, SA: Equilibrium programming in Hilbert spaces. J Nonlinear Convex Anal. 6, 117-136 (2005)

9. Takahashi, S, Takahashi, W: Viscosity approximation methods for equilibrium problems and fixed point problems in Hilbert spaces. J Math Anal Appl. 331, 506-515 (2006)

10. Su, Y, Shang, M, Qin, X: An iterative method of solutions for equilibrium and optimization problems. Nonlinear Anal. 69 , 2709-2719 (2008). doi:10.1016/j.na.2007.08.045

11. Tada, A, Takahashi, W: Weak and strong convergence theorems for a nonexpansive mapping and an equilibrium problem. J Optim Theory Appl. 133, 359-370 (2007). doi:10.1007/s10957-007-9187-z

12. Plubtieng, S, Punpaeng, R: A new iterative method for equilibrium problems and fixed point problems of nonexpansive mappings and monotone mappings. Appl Math Comput. 197, 548-558 (2008). doi:10.1016/j.amc.2007.07.075

13. Chang, SS, Joseph Lee, HW, Chan, CK: A new method for solving equilibrium problem, fixed point problem and variational inequality problem with application to optimization. Nonlinear Anal. 70, 3307-3319 (2009). doi:10.1016/j. na.2008.04.035

14. Yao, YH, Liou, YC, Yao, JC: Convergence theorem for equilibrium problems and fixed point problems of infinite family of nonexpansive mappings. Fixed Point Theory Appl 12 (2007). Article ID 64363

15. Ceng, LC, Yao, JC: Hybrid viscosity approximation schemes for equilibrium problems and fixed point problems of infinitely many nonexpansive mappings. Appl Math Comput. 198, 729-741 (2007)

16. Colao, V, Marino, G, Xu, H-K: An iterative method for finding common solutions of equilibrium and fixed point problems. J Math Anal Appl. 344, 340-352 (2008). doi:10.1016/j.jmaa.2008.02.041

17. Iusem, AN, Sosa, W: Iterative algorithms for equilibrium problems. Optimization. 52, $301-316$ (2003). doi:10.1080/ 0233193031000120039

18. Nguyen, TTV, Strodiot, JJ, Nguyen, VH: A bundle method for solving equilibrium problems. Math Program Ser B. 116, 529-552 (2009). doi:10.1007/s10107-007-0112-x

19. Ceng, LC, Al-Homidan, S, Ansari, QH, Yao, JC: An iterative scheme for equilibrium problems and fixed point problems of strict pseudo-contraction mappings. J Comput Appl Math. 223, 967-974 (2008)

20. Takahashi, W, Shimoji, K: Convergence theorems for nonexpansive mappings and feasibility problems. Math Comput Model. 32, 1463-1471 (2000). doi:10.1016/50895-7177(00)00218-1

21. Shimoji, K, Takahashi, W: Strong convergence to common fixed points of infinite nonexpansive mappings and applications. Taiwanese J Math. 5(2):387-404 (2001)

22. OHaraa, JG, Pillay, P, Xu, HK: Iterative approaches to finding nearest common fixed points of nonexpansive mappings in Hilbert spaces. Nonlinear Anal. 54, 1417-1426 (2003). doi:10.1016/50362-546X(03)00193-7

23. Nadezhkina, N, Takahashi, W: Strong convergence theorem by a hybrid method for non-expansive mappings and Lipschitz-continuous monotone mappings. SIAM J Optim. 16(4):1230-1241 (2006). doi:10.1137/050624315

24. Opial, Z: Weak convergence of the sequence of successive approximation for nonexpansive mappings. Bull Am Math Soc. 73, 561-597 (1967)

25. Rockafellar, RT: On the maximality of sums of nonlinear monotone operators. Trans Am Math Soc. 149, 75-88 (1970). doi:10.1090/S0002-9947-1970-0282272-5

26. Ceng, LC, Hadjisavvas, N, Wong, NC: Strong convergence theorem by a hybrid extragradient-like approximation method for variational inequalities and fixed point problems. J Global Optim. 46, 635-646 (2010) (2010). doi:10.1007/s10898-0099454-7

27. Bruck, RE: A simple proof of the mean ergodic theorem for nonlinear contractions in Banach spaces. Israel J Math. 32, 107-116 (1979). doi:10.1007/BF02764907

28. Bruck, RE: On the convex approximation property and the asymptotic behavior of nonlinear contractions in Banach spaces. Israel J Math. 38, 304-314 (1981). doi:10.1007/BF02762776

doi:10.1186/1687-1812-2011-12

Cite this article as: Peng: Some extragradient methods for common solutions of generalized equilibrium problems and fixed points of nonexpansive mappings. Fixed Point Theory and Applications 2011 2011:12.

\section{Submit your manuscript to a SpringerOpen ${ }^{\odot}$ journal and benefit from:}

- Convenient online submission

Rigorous peer review

- Immediate publication on acceptance

- Open access: articles freely available online

- High visibility within the field

- Retaining the copyright to your article

Submit your next manuscript at $\gg$ springeropen.com 